\title{
Screen printed electrodes based on polymer/ MWCNT and polymer/G nanocomposite for advanced gas sensing application
}

\begin{abstract}
This paper reported the results of application of screen printed electrodes (SPE) based on polymer/carbon nanostructures (multi wall carbon nanotubes - MWCNT and grapheme - G) for gas sensors. Commercial SPEs were modified by polymers such as conductive polymer Poly Vinylidene Fluorid - PVDF and biocompatible Poly Ethylene Glycol - PEG. Modified SPEs were tested in ammonia vapors with different concentration: 3, 6.2, 12.5 and $25 \%$ (wt.). Sensor-testing was performed by monitoring the change in the electrical resistance of the electrodes. The physical characterization of the sensing electrodes was performed by Scanning electron microscopy (SEM) and Fourier transform infrared spectroscopy (FTIR) in ATR mode. The surface morphology of CNT/PEG and CNT/ PVDF modifications, after coating with $25 \% \mathrm{NH} 3$, expressed the effects of ammonia on the surface of the nanocomposite layer. The ammonia solution acts aggressively on the modified surface, causing furrows to form in the uniform structure and very small nanorods of oxides.
\end{abstract}

Keywords: gas sensor, MWCNT, graphene, screen printed electrode
Volume 4 Issue 4 - 2020

\author{
Anita Grozdanov, Iva Dimitrievska, Perica \\ Paunović, Aleksandar Petrovski \\ Department of Material Science and Nanotechnology, Ss Cyril \\ and Methodius University, Republic of Macedonia \\ Correspondence: Anita Grozdanov, Department of Material \\ Science and Nanotechnology, Ss Cyril and Methodius University, \\ Rugjer Boskovic 16, 1000 Skopje, Republic of Macedonia, \\ Tel 0038975303578,Email anita.grozdaov@yahoo.com
}

Received: July 22, 2020 | Published: August II, 2020

\section{Introduction}

The atmospheric air we breathe daily contains numerous types of chemical particles, both natural and artificial, some of which are vital to our lives, while many others are more or less harmful. Vital gases, such as oxygen and moisture, should be kept at appropriate levels in the atmosphere in which we live, while hazardous should be controlled to be below the designated levels. For toxic gases, unpleasant odors, volatile organic compounds (VOCs) and other air pollutants, their standards are regulated by several laws based on the intensity of the toxicity or the discomfort of each gas. Atmospheric pollution can cause major disaster in a short period of time, as this type of pollution can spread rapidly over large areas. To prevent or minimize the damage caused by atmospheric pollution, monitoring and control systems are needed that can quickly and reliably detect and quantify sources of pollution within the range of regulatory standard values. Successful implementation of such a system is enabled using gas sensors. Nanostructures, among them carbon nanotubes (CNTs), have received a huge attention last years in various fields because of their enormous potential and their unique structural and electronic properties. ${ }^{1-3} \mathrm{CNTs}$ exhibit interesting electrochemical behavior, especially modified ones due to the presence of reactive groups on the surface. ${ }^{1,4}$ Compton's group presented a work about the reason why CNTs exhibit the enhanced electrolytic activity and suggested that this activity is due to the presence of edge-plane like sites located at the and in the "defect" areas of the tubes ${ }^{5}$ So, in general, lower overvoltage's and higher peak currents were observed in the voltammetry response of several molecules at electrodes with modified CNTs. Due to these unique properties, CNTs have received a great attention for preparation of electrochemical gas sensors. ${ }^{6-9}$ At the beginning, some pre-treatments of the CNTs are necessary to eliminate metallic impurities and to allow further functionalization of carbon surface. Mainly, most of the protocols are based on the oxidation of CNTs under different conditions when the ends and sidewalls become rich in oxygenated - carboxylic groups. ${ }^{8-10}$
Sin $\mathrm{M}$ et al. ${ }^{11}$ have found that the electronic sensitivity of CNTs to various target gases is highly limited by the binding energy and charge transfers of the molecules with the CNTs wall. When they were functionalized, active bonds appear on the side walls of CNTs which would indeed enhance their sensing capability towards wide range of gaseous vapors. ${ }^{11}$ Activation by treatment in acidic solutions (sulphuric, nitric, hydrochloric) was widely used. ${ }^{4,6}$ Also, in some cases, various pre-treatments were designed based on a combination of different chemical and electrochemical procedures. ${ }^{6,10}$ Because of the insolubility of CNTs in usual solvents, several strategies were proposed for the immobilization of CNTs on electrochemical transducers, like dispersion in polyelectrolytes or incorporation in polymer composites using as a distinct binders. ${ }^{10-13}$ Zhang et $\mathrm{al}^{12}$ performed an electrochemical functionalization of CNT with polyaniline. ${ }^{12}$ On-line detection of $\mathrm{NH}_{3}$ was obtained by the PANICNT network based sensors. Its sensitivity was up to $2.44 \% \Delta \mathrm{R} / \mathrm{R}$ per ppm $\mathrm{NH}_{3}$, which was 60 times more than pristine CNTs. The recovery time was in range of hours, the response time was in range of minutes, while the sensitivity was high at low temperature. The main goal of this paper is to obtain nanocomposite-based sensors from biocompatible polymer - polyethylene glycol (PEG) and conductive polymer - Poly Vinylidene fluoride (PVDF) reinforced with carbon nanostructures designed for the detection of gas pollutants. The subject of research was a modification and application of nanocomposites using the drop method (drop modification method), their characterization, performance of testing of gaseous sensing electrodes in alkaline vapours $\left(\mathrm{NH}_{3}\right)$ with different concentration.

\section{Materials and methods}

Experimental work in this research was carried out in three phases. The first phase was followed by the change in the resistance of the commercial electrodes, by exposing the sensor to ammonia vapor's of various concentrations. Commercially available, screen printed carbon electrodes (SPEs), based on MWCNT (DRP 110) and G 
(DS1100), with carbon working electrode of $4 \mathrm{~mm}$ diameter, were ordered from Dropsens, Spain. In the second phase, the polymers (Poly Ethylene glycol (PEG) and Poly- Vinylidene fluoride (PVDF)) were applied by the drop modification method on the sensor electrode and the change in resistance of the sensor to exposure to ammonia vapor with varying concentration is monitored again. In the third phase, the characterization of the nanocomposite film was performed after the sensor measurements. In the first phase, the performances of commercial sensor electrodes (PANI, CNT, G) in ammonia vapors with different concentration: $3,6.2,12.5$ and $25 \%$ (wt.) were tested. In fact, the testing was performed by monitoring the change in the electrical resistance of the electrodes. Carbon sensor electrodes require some external excitation such as thermal heating or electric pulse, so the sensing activity of the electrodes was started to be monitored by heating the ammonia solution and evaporating it around $50^{\circ} \mathrm{C}$. Experimental scheme for electrochemical measurements is shown in Figure 1.

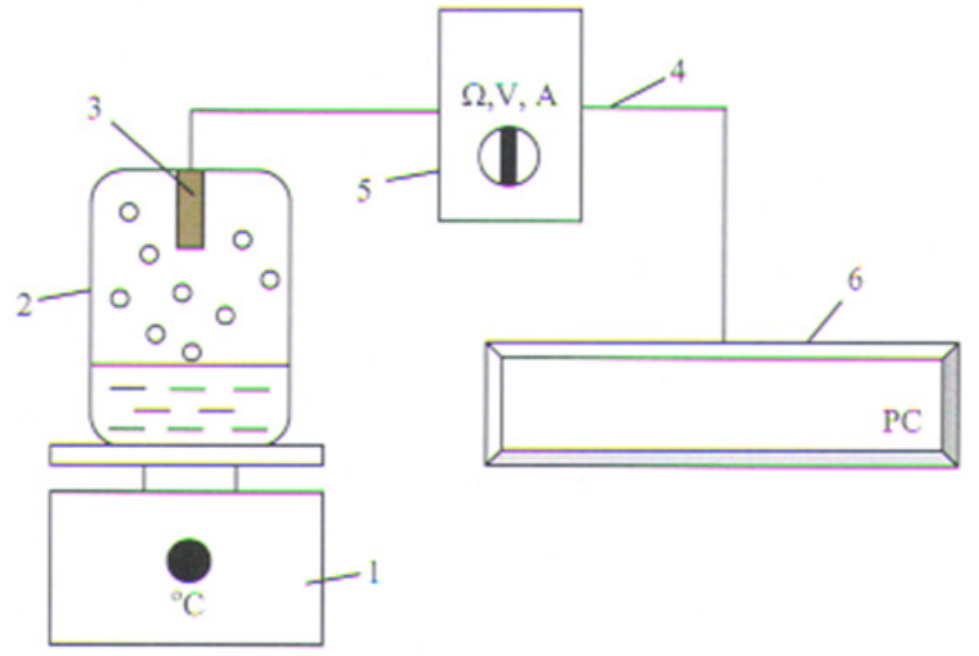

Figure I Experimental scheme of SPE-testing.

I. Magnetic stirrer; 2. Tube with NH3; 3. SPE electrode; 4. Cable; 5. Multi Meter; 6.PC Computer.

In the second phase, the synthesis of the nanocomposite film was performed using the drop method, i.e. drop modification method, where conductive polymer (PVDF) (PVDF dissolved in n-dimethyl acetamide at $2 \%$ concentration) and biocompatible polymer (PEG) (PEG 400 - used commercial type of low-molecular-weight of 400g/ mol) were deposited to the surface of commercial sensors (CNT, $\mathrm{G})$.The prepared polymers, using a thin medical needle, were applied to the sensor surface of the commercial electrodes (CNT, G) (Figure 2). After application, the polymer modified SPE were dry at room temperature. A total of four types of carbon SPE-based sensors were prepared: 1. CNT + PVDF; 2. CNT + PEG 400; 3. G + PVDF; 4. G + PEG 400. In the third phase, the physical characterization of the sensing electrodes, which were charged with ammonia, was performed. The characterization was performed by Scanning electron microscopy (SEM) and Fourier transform infrared spectroscopy (FTIR) in ATR mode. Surface morphology of the SPE electrodes was analyzed by VEGAJ TESCAN scanning electron microscope, using a secondary electron detector and acceleration voltage of $20 \mathrm{kV}$. FTIR-ATR spectra were collected by Perkin Elmer-Spectrum 100 machine at 64 scans, in ATR mode in the range from $400 \mathrm{~cm}^{-1}$ to $4000 \mathrm{~cm}^{-1}$ Figure 2.

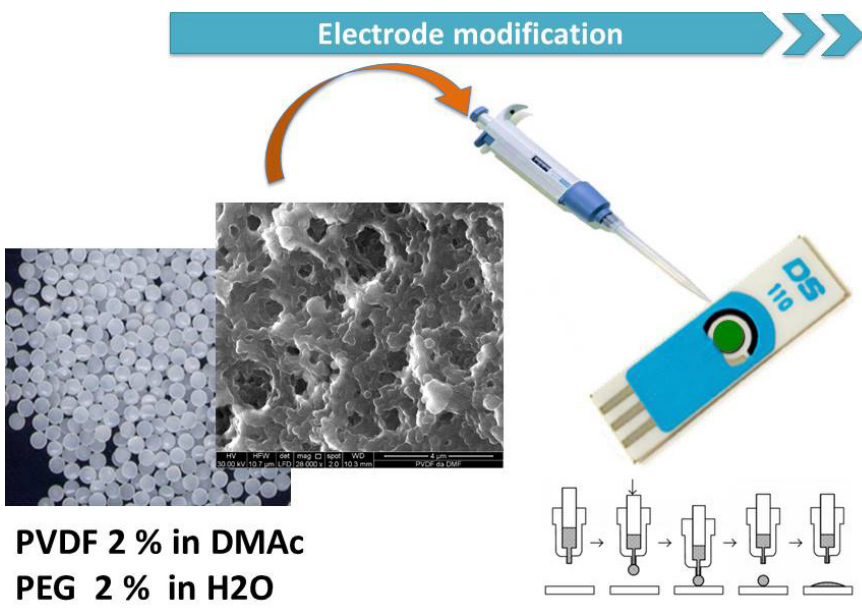

Carbon electrodes were modified with PEG and PVDF solutions

- Solvent has been dried in air and then under vacuum

Figure 2 Modification of screen printed electrodes. 


\section{Results and discussion}

In order to obtain the best analytical response of SPE - sensors on different concentration of $\mathrm{NH}_{3}$, SPEs-electrodes based on electro conductive PANI polymer were compared with SPEs with two carbon nanostructures, $\mathrm{CNT}$ and $\mathrm{G}$ (Figure 3 ). In the first phase the performance of the sensors was tested by monitoring the change in electrical resistance. As a result of the pairing, resistance change is expected i.e. conductivity of electrodes. All of the obtained characteristic curves confirm that over time, at all concentrations, the resistance decreases, and the conductivity increases. The least resistance, i.e. carbon nanotubes shows the highest conductivity. Electrodes made of carbon structures, such as CNT and G, show higher conductivity than PANI electrodes. The reason for this is the electrical nature of carbon structures that possess much better electrical properties than polymers, i.e. PANI in this case. It was evident that for all studied SPEs based sensors, the measured data suggested that it took 6-10 min to reach equilibrium (Figure 3A-3C). In the second phase, the sensor performance was again tested, but this time on modified nanocomposite electrodes. In this case too, a change in resistance is expected due to the varying sensitivity of the polymer film applied to the nanostructured portion of the electrode. All curves show that resistance decreases over time, and the conductivity increases, as was the case with commercial sensors. PVFD and PEG modified SPEnanocomposite sensors have higher conductivity than pure ones. The best performance is the CNT/PVDF combination, due to the synergy between carbon nanostructure and polymer film sensitivity. CNT/PEG modification also shows excellent conductivity, although PEG does not belong to the conductive polymers group, it has high sorption power and the ability to bar and thereby pressurize carbon nanotubes and modify the properties of the entire sensor. G/polymer modifications have lower solubility than CNT/polymer. The G/PVDF has better results due to the electrical properties of the polymer, while the G/PEG shows the lowest conductivity at all ammonia concentrations. Comparison diagrams for resistivity changes for all studied SPE sensor electrodes are shown in Figure 4- Figure 6.

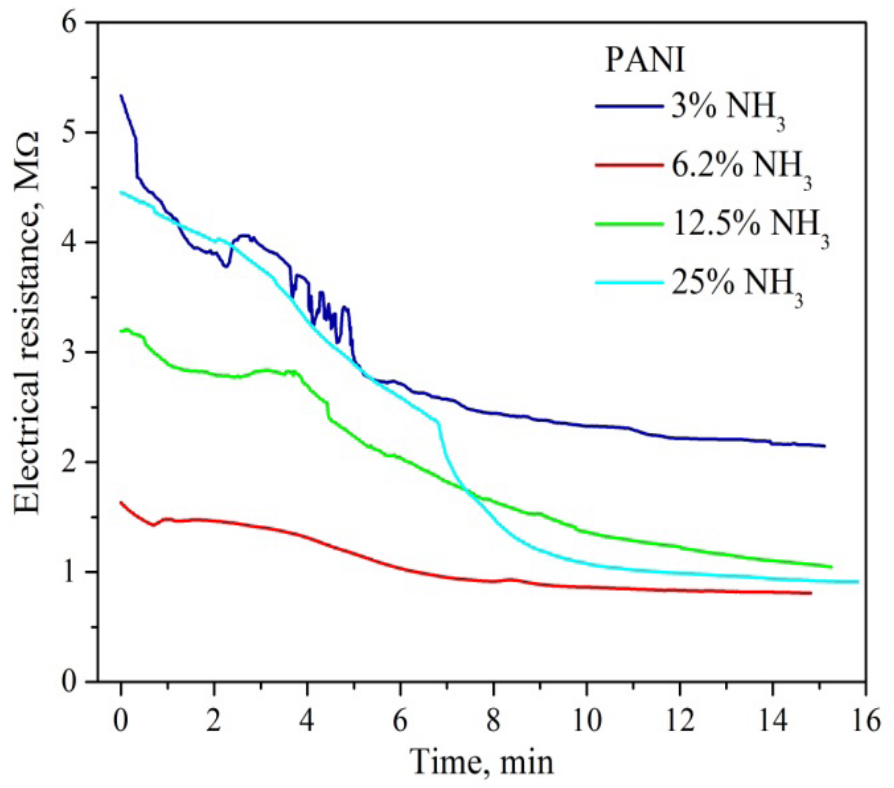

(A)

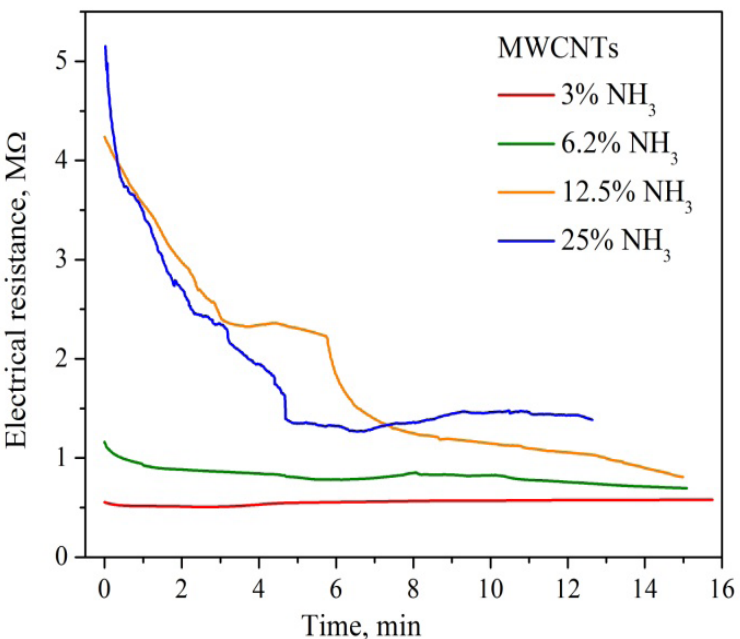

(B)

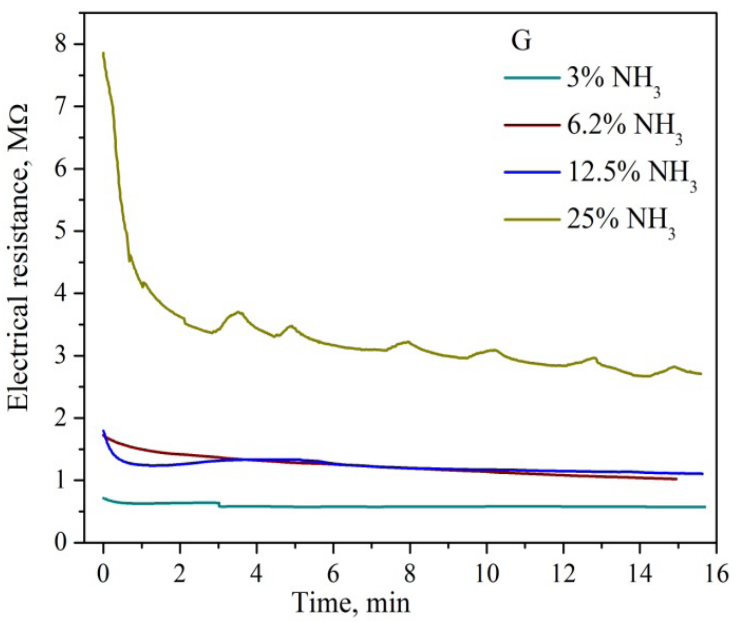

(C)

Figure 3 Resistivity changes of pure PANI (3A), MWCNT (3Bb) and G (3C) - SPE in various concentration of $\mathrm{NH}_{3}$ ions.

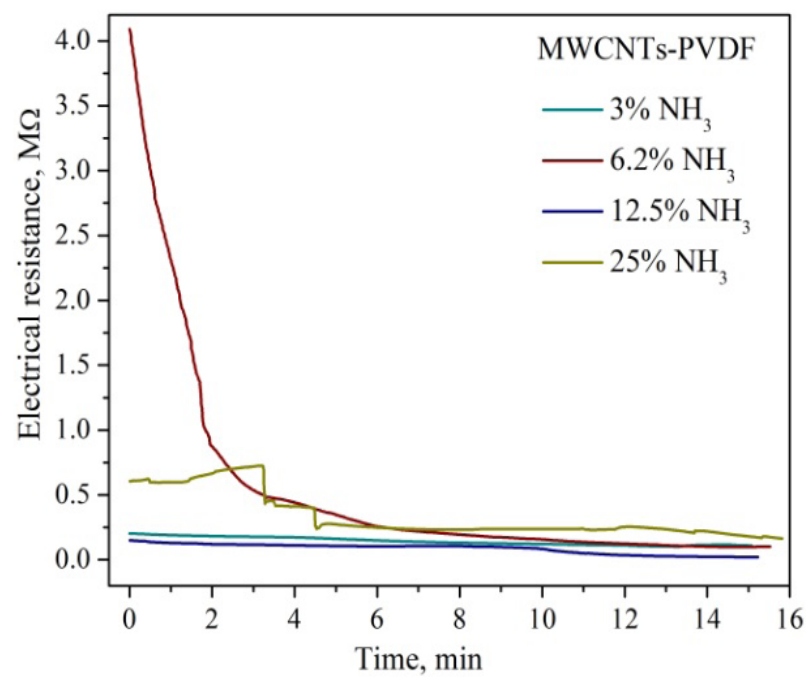

(A) 


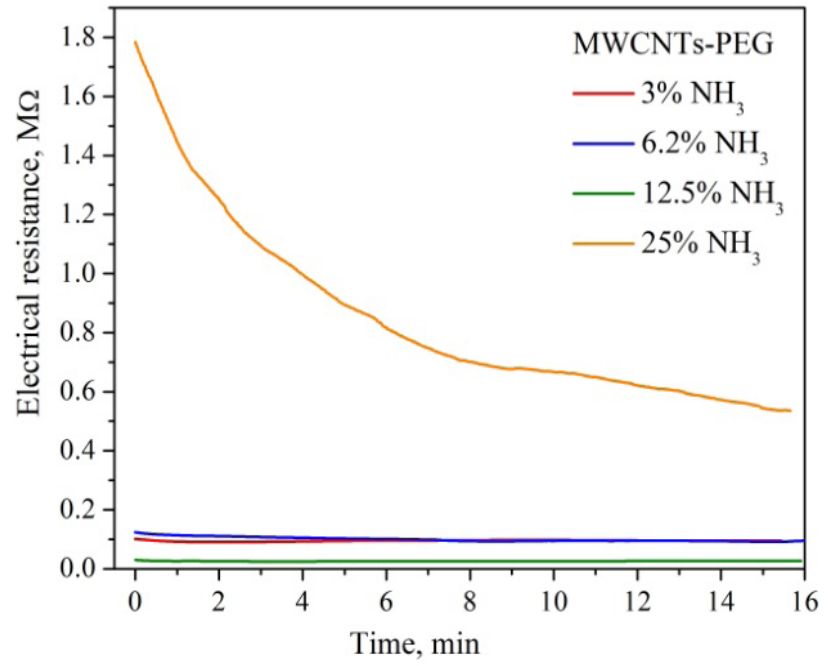

(B)

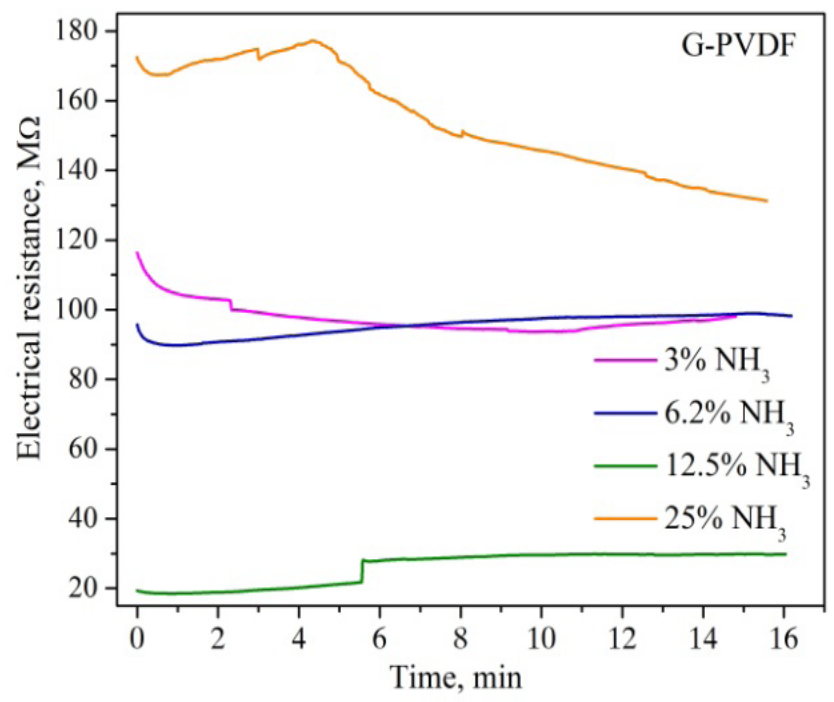

(C)

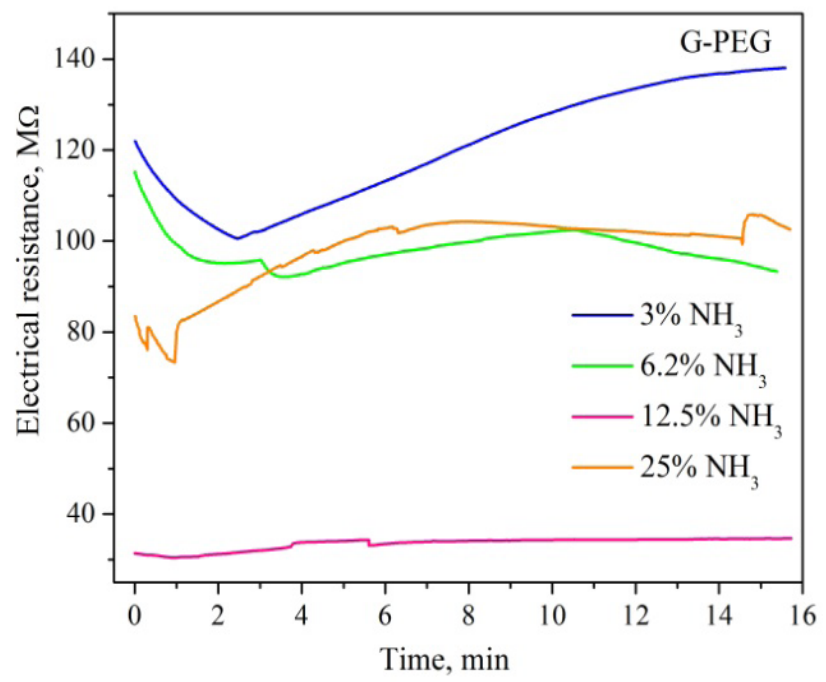

(D)

Figure 4 Resistivity changes of PVDFand PEG modified MWCNT (4A, 4B) and $G$ SPE $(4 C, 4 D)$ in various concentration of $\mathrm{NH}_{3}$ ions.

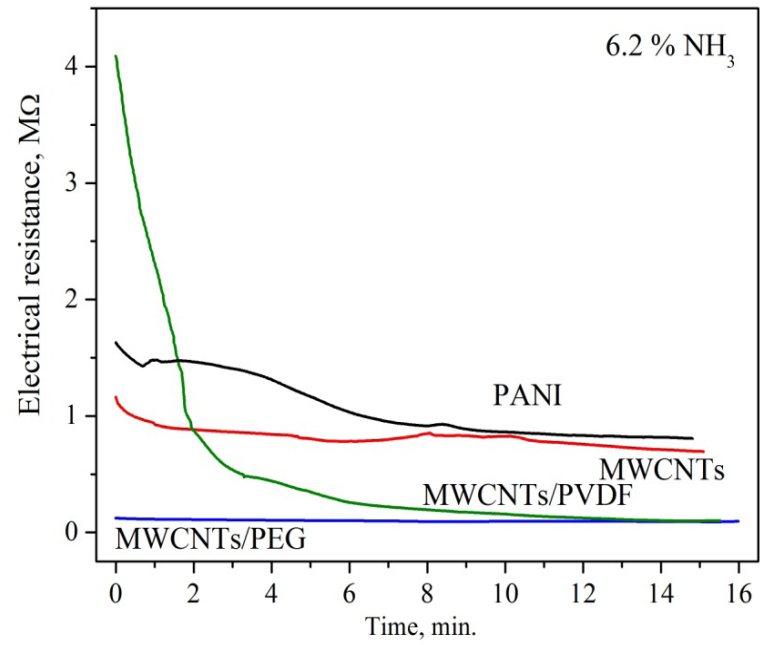

(A)

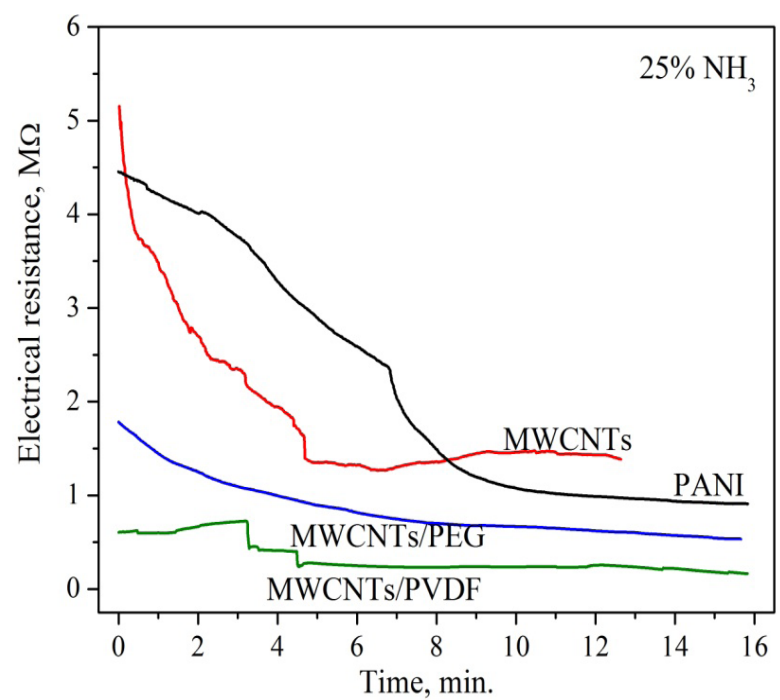

(B)

Figure 5 Comparison of resistivity changes of MWCNT-based SPEs sensors for $6 \%(5 \mathrm{~A})$ and $25 \%(5 \mathrm{~B})$ of $\mathrm{NH}_{3}$ ions.

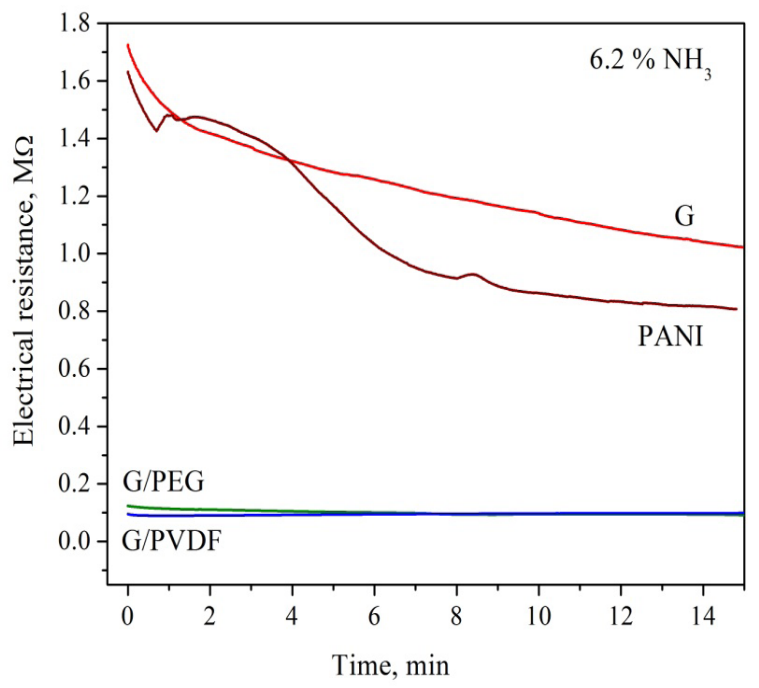

(A)

Citation: Grozdanov A, Dimitrievska I, Paunović P, et al. Screen printed electrodes based on polymer/MWCNT and polymer/G nanocomposite for advanced gas sensing application. Material Sci \& Eng. 2020;4(4):I02-I08. DOI: I0.I5406/mseij.2020.04.00I35 


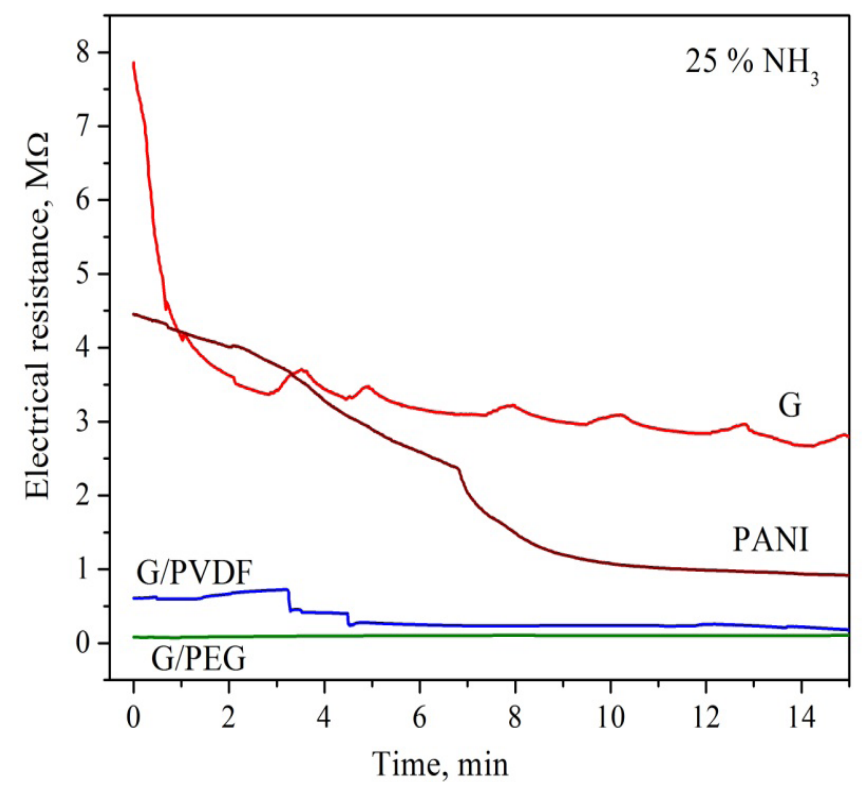

(B)

Figure 6 Comparison of resistivity changes of G-based SPEs sensors for $6 \%$ (6A) and $25 \%$ of $\mathrm{NH}_{3}(6 \mathrm{~B})$ ions.

In order to compare the sensitivity of MWCNT-based SPE on alkaline and acidic medium, we have compared the resistivity changes of MWCNT-based SPE on $3 \% \mathrm{NH}_{3}$ with that one on $3 \%$ $\mathrm{H}_{2} \mathrm{SO}_{4}$ vapours. ${ }^{13}$ Characteristic curves are shown in Figure 7. Lower resistivity i.e. higher conductivity was obtained for acidic medium of $\mathrm{H}_{2} \mathrm{SO}_{4}$ vapor's Figure 7. Due to the ammonia treatment of the SPEs-sensor electrodes, morphological changes occurred on the nanocomposite surface. Morphological changes of SPEs sensors were followed by SEM and they are shown in Figure 8. Due to the alcaline - ammonia treatment, small nanorods of salt structures were found in CNT-based electrodes and these salt's nanorods could be responsible for the increased conductivity. The obtained SEM images clearly show the abrasive effects caused by ammonia on the surface of the nanocomposite layer of the coupled CNT/PEG electrode (Figure 8B). The ammonia solution acts aggressively on the modified surface, causing furrows to form in the uniform structure. The microphotographs also show ammonia corrosion on the surface of the CNT/PVDF nanocomposite layer (Figure 8A). It causes the formation of abrasive channels and deviations from the uniformity of the structure as well as very small nano-rods of oxides. Using FTIR-ATR, the changes that have occurred on the surface of the nanostructure during the absorption of ammonia were observed. The obtained FTIRATR spectra for CNT/PVDF SPEs-sensors and for CNT/PEG SPEs sensors are shown in Figure 9. As can be seen from the spectra of CNT/PEG and CNT/PVDF modifications, the wavelengths on which the peaks appear are shifted, as a result of the change in the amount of donor and acceptor groups on the surface of the carbon nanostructure. The peaks at 1292 and $1236 \mathrm{~cm}^{-1}$ correspond to the C-Nstretching vibration. The peaks at 1131 and $800 \mathrm{~cm}^{-1}$ were attributed to in-plane bending of $\mathrm{C}-\mathrm{H}$ and the out-of-plane bending of $\mathrm{C}-\mathrm{H}$, respectively. ${ }^{12}$ Also, It was found that due to the ammonia vaporization, the intensity of the peaks at 890 and $1150 \mathrm{~cm}^{-1}$ decreased, while the intensity of the peaks at $1380 \mathrm{~cm}^{-1}$ increased, as result of the increasing of the ammonia concentration Figure 9.

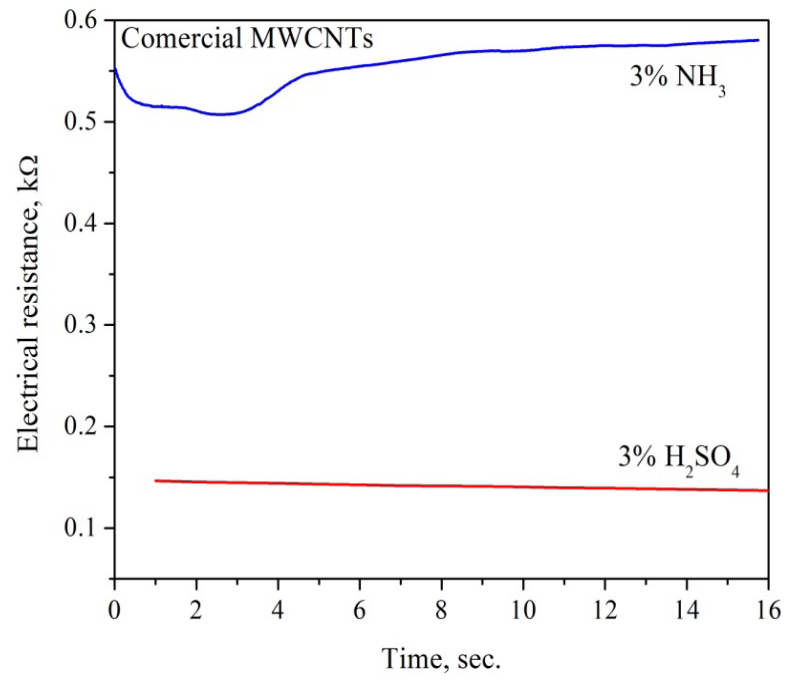

Figure 7 Comparison of resistivity changes of MWCNT-based SPEs sensors for $3 \% \mathrm{NH}_{3}$ ions and $3 \% \mathrm{H}_{2} \mathrm{SO}_{4}$ ions.

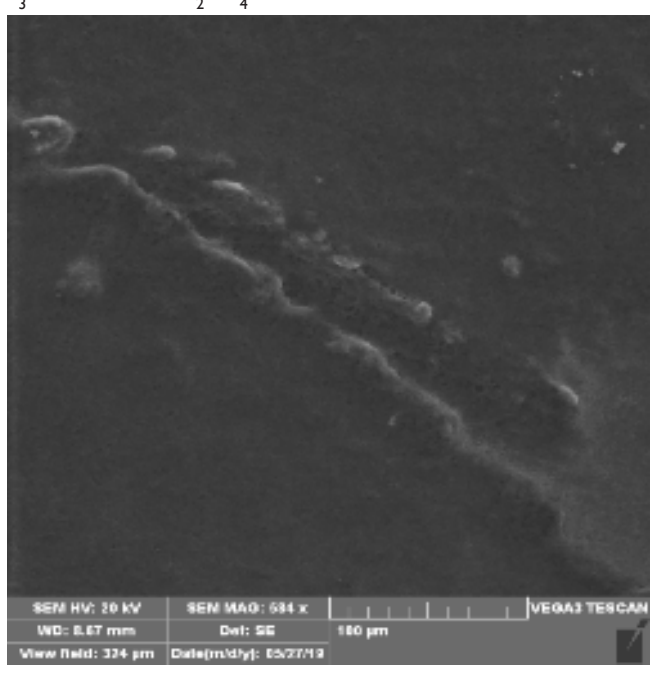

(A)

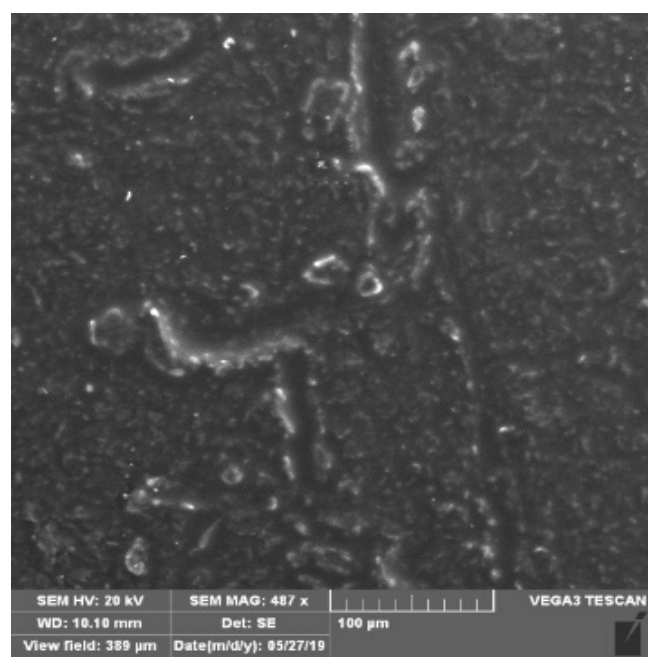

(B)

Figure 8 SEM Morphology of CNT / PVDF electrode - 25\% NH3 (3A) and CNT / PEG electrode - $25 \% \mathrm{NH}_{3}(3 \mathrm{~B})$. 


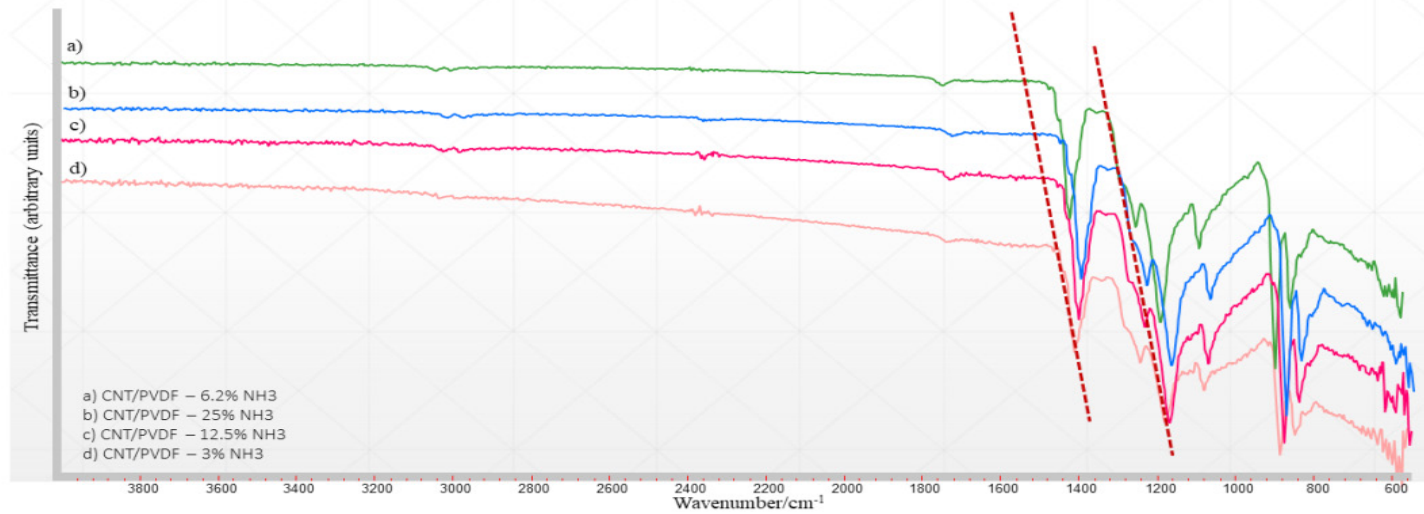

(A)

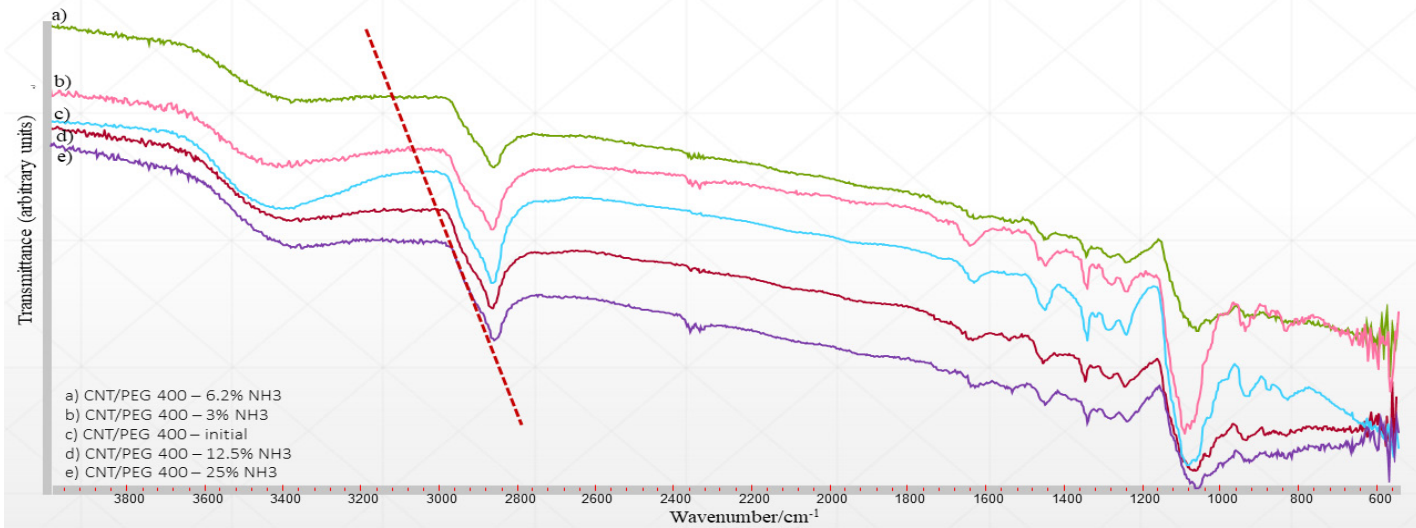

(B)

Figure 9 FTIR spectroscopy of CNT/PVDF (9A) and CNT/PEG (9B) ammonia treated electrodes.

\section{Conclusion}

In this work, gas sensors with surface modification of commercial electrodes (CNT, G) were obtained. The commercial electrode modification was performed by the drop modification method, where a thin conductive polymer film (PVDF) and biocompatible polymer (PEG) were applied to the electrode surface. A total of four systems have been developed: CNT / PVDF; CNT / PEG; G / PVDF; G / PEG. The obtained SPEs- gas sensors were tested to detect ammonia vapors in the atmosphere. In order to compare the results obtained after modification of the electrodes, the performance of commercial electrodes (CNT, G, PANI) was first tested by monitoring the changes in electrical resistance. This was done under conditions of oxidation i.e. at the electrode coupling with different concentrations of ammonia vapor. Best sensor activity: least resistance, i.e. the highest conductivity was observed for CNT- based electrodes. After modification performance testing has again been performed, but this time on modified nanocomposite electrodes, by monitoring the change in electrical resistance, at the same conditions as before. Although all modifications show excellent electrical capabilities, the best conductivity is shown by CNT / PVDF modification. In fact, it is due to the synergy between carbon nanostructure and polymer film sensitivity. Excellent results are also shown by PEG modifications. Although PEG does not belong to the conductive polymer group, the swollen polymer film puts pressure on the carbon nanotubes present in the structure and changes the properties of the entire sensor.

The characterization of the modified nanocomposite electrodes was performed by SEM microscopy and Fourier transform infrared spectroscopy (FTIR). The surface morphology of CNT/PEG and CNT/PVDF modifications, after coating with $25 \% \mathrm{NH}_{3}$, was analyzed using SEM photographs. In both modifications, the effects of ammonia on the surface of the nanocomposite layer can be observed. The ammonia solution acts aggressively on the modified surface, causing furrows to form in the uniform structure and very small nano-rods of oxides. Using FTIR-ATR spectroscopy, some of the changes that have occurred on the surface of the nanostructure during the absorption of ammonia were confirmed. As can be seen from the spectra of CNT/PEG and CNT/PVDF SPEs modified electrodes, the wavelengths on which the peaks appear are shifted, as a result of the 
change in the amount of donor and acceptor groups on the surface of the carbon nanostructure. The general conclusion of this work is that the modified sensor electrodes exhibit excellent electrical properties, are characterized by high conductivity and have great potential application in the field of gas pollutants detection.

\section{Acknowledgments}

The research reported in this paper was financially supported by the bilateral scientific project between Faculty of Technology and Metallurgy - University Ss Cyril and Methodius in Skopje from Republic of North Macedonia and Montanuniversität Leoben Department Kunststofftechnik from Austria.

\section{Conflicts of interest}

Authors declare that there is no conflict of interest.

\section{References}

1. Wang Y, Yeow JTW. A review of carbon nanotubes-based gas sensors. $J$ Sensors. 2009;1-24:493904.

2. Li J, Lu Y, Ye Q, et al. Carbon nanotubes sensors for gas and organic vapor detection. Nano Lett. 2003;3(7):929-933.

3. Zhao J, Buldum A, Han J, et al. Gas molecule adsorption in carbon nanotubes and nanotube bundles. Nanotechnology. 2002;13(2):195-200.

4. Ueda T, Katsuki S, Takahashi K, et al. Fabrication and characterization of carbon nanotube based high sensitive gas sensors operable at room temperature. Diam Relat Mater. 2008;17(710):1586-1589.
5. Banks CE, Davies T, Wildgoose GG, et al. Electrocatalysis at Graphite and Carbon Nanotube Modified Electrodes: Edge-Plane Sites and Tube Ends Are the Reactive Sites. Chem Commun. 2005;36(7):829-841.

6. Grozdanov A, Petrovski A, Avella M, et al. Spectroscopically Study of Nanocomposites Based on PANI and Carbon Nanostructures for $\mathrm{pH}$ Sensors, Bulgarian Chemical Communications. 2019;51Special Issue D:36-41.

7. Tomova A, Gentile G, Grozdanov A, et al. Multinanosensors Based on MWCNTs and Biopolymer Matrix - Production and Characterization. Acta Physica Polonica Series A. 2017;132(4):1251-1255.

8. Kim S. CNT sensors for detecting gases with low adsorption energy by ionization. Sensors. 2006;6(5):503-513.

9. Azar MT, Xie Y. Sensitive $\mathrm{NH}_{3} \mathrm{OH}$ and $\mathrm{HCl}$ gas sensors using selfaligned and self-welded multiwalled carbon nanotubes. IEEE Sens J. 2007;7(10):1435-1439.

10. Petrovski A, Paunović P, Grozdanov A, et al. Electrochemical Synthesis of PANI/GrapheneNanocomposites Aimed for Sensors. Advanced Nanotechnologies for Detection and Defence against CBRN Agents. 2018:221-227.

11. Sin MLY, Chow GCT, Wong GMK, et al. Ultralow-power alcohol vapor sensors using chemically functionalized multiwalled carbon nanotubes. IEEE Trans Nano technol. 2007;6(5):571-577.

12. Zhang T, Nix MB, Yoo BY, et al. Electrochemically Functionalized SingleWalled Carbon Nanotube Gas Sensor. Electroanalysis. 2006;18:1153.

13. Chepishevski G, Petrovski A, Grozdanov A, et al. MWCNT/PANI Screen Printed Electrodes for Gas Sensors. Advanced Nanotechnologies for Detection and Defence against CBRN Agents. 2018;389-396. 